Document downloaded from:

http://hdl.handle.net/10251/56670

This paper must be cited as:

Almerich Chulia, AI.; Fenollosa Forner, EJ.; Cabrera Fausto, I. (2015). GFRP Bar:

Determining Tensile Strength with Bending Test. Advanced Materials Research. 1083:90-96. doi:10.4028/www.scientific.net/AMR.1083.90.

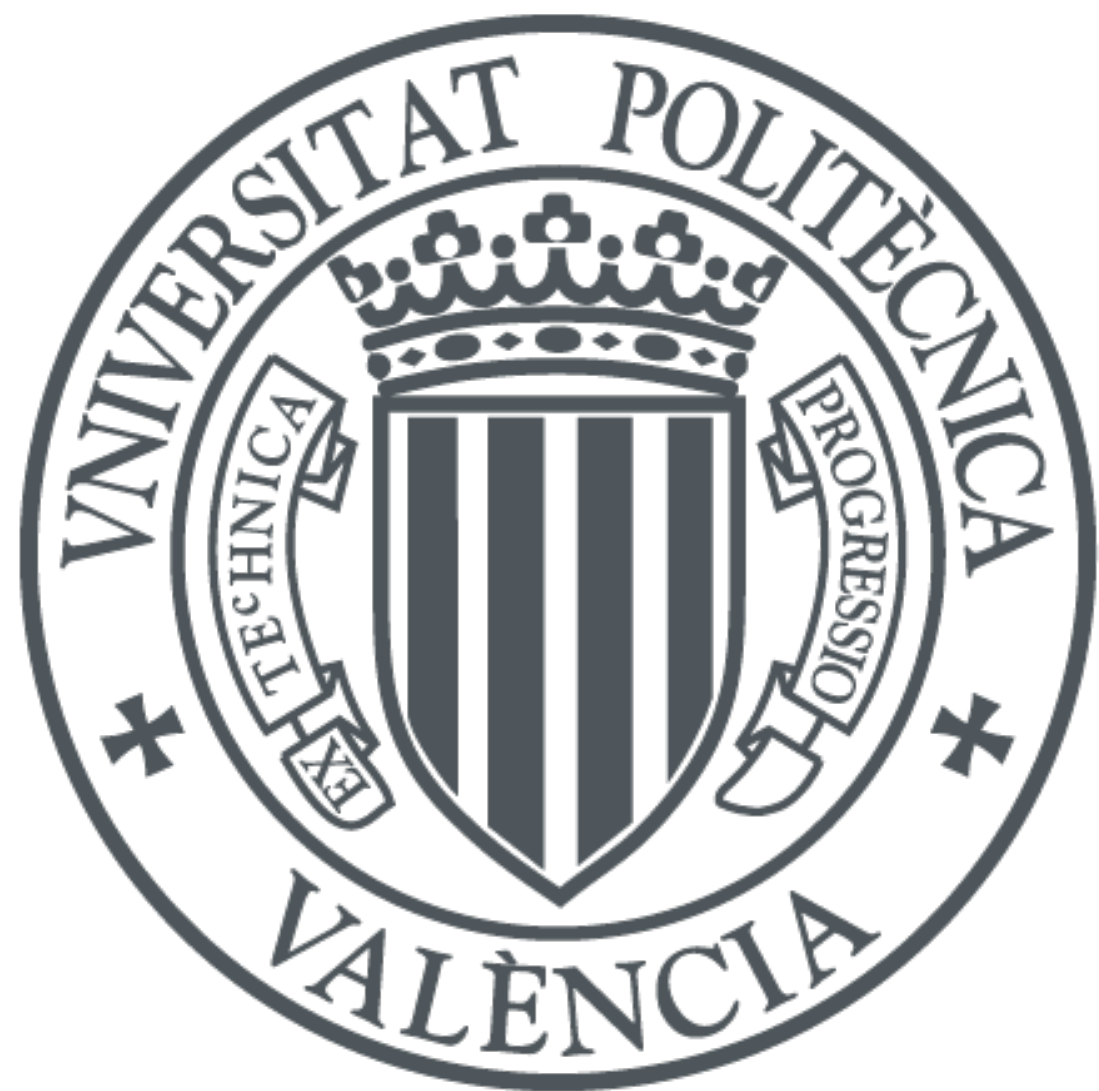

The final publication is available at

http://dx.doi.org/10.4028/www.scientific.net/AMR.1083.90

Copyright Trans Tech Publications

Additional Information 


\title{
GFRP bar: Determining tensile strength with bending test.
}

\author{
A. Almerich ${ }^{1, a}$, E. Fenollosa ${ }^{1, b}$ and I. Cabrera ${ }^{1, c}$ \\ ${ }^{1}$ Department of Continuum Mechanics and Theory of Structures \\ Universitat Politècnica de València \\ Camí de Vera s/n, 46022 Valencia, Spain \\ aanalchu@mes.upv.es, befenollo@mes.upv.es, 'ivcabfau@mes.upv.es
}

Keywords: GFRP bar, strength, tension, bending

\begin{abstract}
In order to obtain GFRP reinforcement bars it is necessary to undertake tests regulated code which require important mechanical tools. This paper presents a method which allows for determining GFRP rebars tensile strength value from their flexural strength value which has been obtained with a simple, inexpensive and reliable test. This method results will be verified by applying it to values obtained in a series of bending tests and comparing these results with values obtained in tensile tests. Values concordance for small diameter GFRP rebars is very good.
\end{abstract}

\section{Introduction}

Glass-fiber reinforced plastic rebars are increasingly used by engineers when designing structures because of their excellent strength properties. This material strength is characterized by its capacity to bear a load without excessive deformation or failure. When a sample of GFRP is tested under axial force, the applied force when divided by the area of the cross-section (stress) is proportional to the ratio between length increase and its initial length (strain). When the applied load is moved away, GFRP gets back to its initial shape and length. In other words, GFRP rebars have linear elastic behavior under axial forces.

Having typified and certified GFRP rebars strength properties [1], it is necessary to determine rebars tensile strength by means of a simple, inexpensive and reliable method which obviates undertaking the laborious tests established in the code.

Not only the lack of regulations and design guides but also the huge variability of applications and the lack of standardization of GFRP components frequently demands carrying out specific experimental programs aimed to confirm theoretical results within particular cases.

For other materials such as concrete, codes consider the possibility of determining tensile stress by means of an indirect tensile test until failure (UNE 83306:1985) [2] or by means of a bending essay (UNE-EN 14651:2007+A1:2008) [3]. Therefore, if guidelines of tests developed for other materials $[2,3]$ are followed, GFRP reinforcement bars tensile strength could be determined by means of similar tests.

The present paper is presented as an experimental procedure to determine GFRP rebars tensile strength by means of a bending test with three points which is much more simple and inexpensive. Procedure's reliability will be verified by means of applying it to the values obtained in a series of bending tests and its comparison with the values obtained in pure tensile tests. Values obtained will be valid as long as tested rebars accomplish certain specifications. 


\section{Tensile strength determining by means of bending test}

Rebars tensile behavior was assessed from flexural strength which was determined considering the load-displacement diagram. Bending test consisted of applying a point load perpendicularly to the specimen axis in its middle point. The bar was simply resting on both ends.

Considering European Codes UNE-EN ISO 178 [4] and UNE-EN 13706-2:2003 [5], in order to determine their flexural strength, specimens to be tested should accomplish the following geometrical ratio between its length between bearing points and the bar diameter.

$$
L=20 \cdot \phi
$$

Being $L$ the length between bearing points and $\phi$ the specimen diameter.

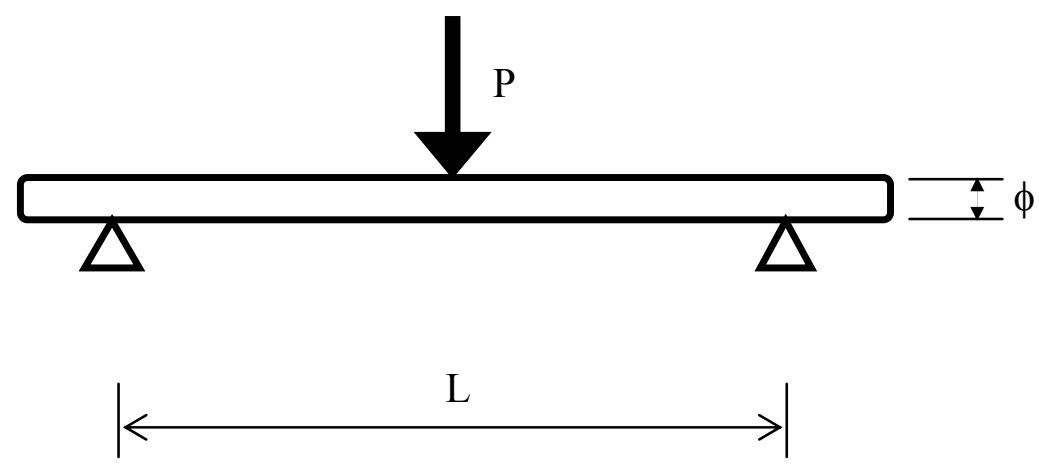

Figure 1. Dynamic test scheme

Bending moment in the bay center produced by a centered point load $\mathrm{P}$ has a maximum value:

$$
M=\frac{P}{2} \cdot \frac{L}{2}
$$

If we assume Navier's hypothesis and accepting that linear behavior is kept until failure then stresses distribution within the cross-section can be obtained by means of the following equation:

$$
\sigma_{\max }=\frac{M}{I} \cdot y=\frac{P \cdot L / 4}{\pi \cdot \phi^{4} / 64} \cdot \frac{\phi}{2}=\frac{8 \cdot P \cdot L}{\pi \cdot \phi^{3}}
$$

Anyhow in order to determine this strength, it will be mandatory to undertake the tensile test established by code whenever the ratio between the applied point load and the tested rebar diameter does not accomplish equation (4):

$$
P \geq 12 \cdot \phi^{2}
$$

\section{Testing plan and results}

Tests were carried out with the laboratory equipment of the Department of Continuum Mechanics and Theory of Structures of the Universitat Politècnica de València. For the three points bending test a universal testing press was employed. This device is certified according to the EN 7500-1 Code by SERVOSIS S.A. and its main characteristics are:

- Specimens are simply resting on both ends on cylindrical metallic pieces.

- Press can apply a maximum load of $98 \mathrm{kN}(10 \mathrm{Tn})$. 
- Point load is applied in the specimen center point by means of an element with a cylindrical head.

- Applied loads and displacements produced are registered by software installed in the press by SERVOSIS S.A.
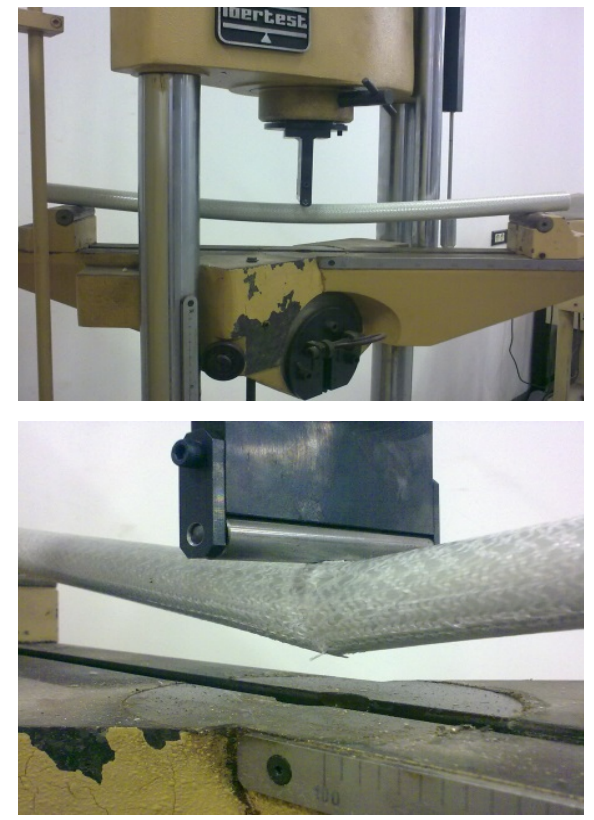
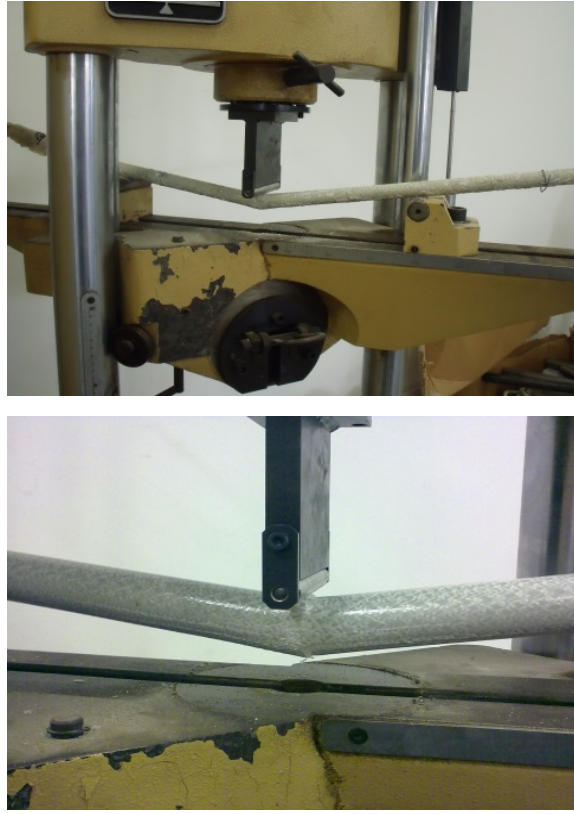

Figure 2. Bending test in order to determine tensile strength

Figure 3 shows a specimen cross-section which has reached failure during the bending essay. It clearly displays compressive fibers and tensile fibers.

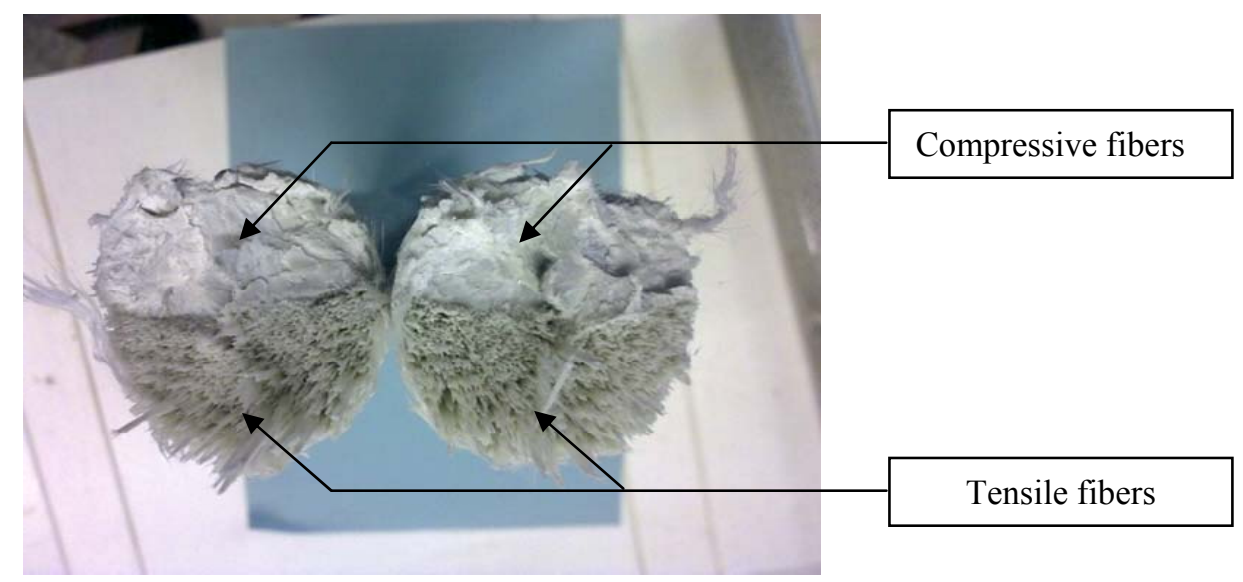

Figure 3. Bending test: compressive and tensile fibers

Bending test has been carried out on a series of specimens and bending failure load has been obtained. Tensile strength will be determined from this value. Results obtained are presented in the following tables for each diameter tested. Nevertheless, an estimator has been used in order to assure a minimum value of the assessed characteristic. It has a confidence level of $95 \%$ as stipulated in UNE 66040:2003 [6].

$$
\sigma_{\text {caract }}=\sigma-\left(1-\delta \cdot t_{1-\alpha}\right)
$$

Being $\sigma$ the arithmetical mean of all values obtained, $\delta$ the quotient between the arithmetic mean and the mean deviation and $\mathrm{t} 1-\alpha$ the coefficient for a confidence level: $\alpha=95 \%$ 
Table 1. Bending test results for diameter $8 \mathrm{~mm}$

\begin{tabular}{c|c|c|c|c|c|c}
$\phi(\mathrm{mm})$ & $\phi$ real $(\mathrm{mm})$ & $\begin{array}{c}\text { Cross Area } \\
\left(\mathrm{mm}^{2}\right)\end{array}$ & $\begin{array}{c}\text { Span } \\
(\mathrm{mm})\end{array}$ & $\begin{array}{c}\text { Pmax } \\
(\mathrm{kN})\end{array}$ & $\begin{array}{c}\text { Deflection } \\
(\mathrm{mm})\end{array}$ & $\begin{array}{c}\sigma \text { max } \\
(\mathrm{MPa})\end{array}$ \\
\hline 8 & 8.00 & 50.27 & 160 & 0.910 & 10.71 & 724.2 \\
\hline 8 & 8.00 & 50.27 & 160 & 0.838 & 12.45 & 666.9 \\
\hline 8 & 8.00 & 50.27 & 160 & 0.871 & 12.12 & 693.1 \\
\hline 8 & 8.00 & 50.27 & 160 & 0.856 & 11.94 & 681.2 \\
\hline 8 & 8.00 & 50.27 & 160 & 0.934 & 11,24 & 743.3 \\
\hline Average & 8.00 & 50.27 & 160 & 0.904 & 10.85 & 719.4 \\
\hline Deviation & 0.00 & & & 0.9 & & 704.7 \\
\hline$\delta$ & $0.0 \%$ & & & 0.03 & & 24.27 \\
\hline Characteristic & 8.00 & & & $3.4 \%$ & & $3.4 \%$ \\
\hline
\end{tabular}

Table 2. Bending test results for diameter $10 \mathrm{~mm}$

\begin{tabular}{c|c|c|c|c|c|c}
$\phi(\mathrm{mm})$ & $\phi$ real $(\mathrm{mm})$ & $\begin{array}{c}\text { Cross Area } \\
\left(\mathrm{mm}^{2}\right)\end{array}$ & $\begin{array}{c}\text { Span } \\
(\mathrm{mm})\end{array}$ & $\begin{array}{c}\text { Pmax } \\
(\mathrm{kN})\end{array}$ & $\begin{array}{c}\text { Deflection } \\
(\mathrm{mm})\end{array}$ & $\begin{array}{c}\sigma \text { max } \\
(\mathrm{MPa})\end{array}$ \\
\hline 10 & 10.00 & 78.54 & 200 & 1.216 & 10.19 & 619.3 \\
\hline 10 & 10.00 & 78.54 & 200 & 1.674 & 15.19 & 852.6 \\
\hline 10 & 10.00 & 78.54 & 200 & 1.560 & 13.16 & 794.5 \\
\hline 10 & 10.00 & 78.54 & 200 & 1.464 & 12.76 & 745.6 \\
\hline 10 & 10.00 & 78.54 & 200 & 1.650 & 15.99 & 840.3 \\
\hline 10 & 10.00 & 78,54 & 200 & 1,482 & 12.79 & 754.8 \\
\hline Average & 10.00 & & & 0.9 & & 767.8 \\
\hline Deviation & 0.00 & & & 0.03 & & 61.29 \\
\hline$\delta$ & $0.0 \%$ & & & $3.4 \%$ & & $8.0 \%$ \\
\hline Characteristic & 10.00 & & & 0.82 & & 644.36
\end{tabular}

Table 3. Bending test results for diameter $12 \mathrm{~mm}$

\begin{tabular}{c|c|c|c|c|c|c}
$\phi(\mathrm{mm})$ & $\phi$ real $(\mathrm{mm})$ & $\begin{array}{c}\text { Cross Area } \\
\left(\mathrm{mm}^{2}\right)\end{array}$ & $\begin{array}{c}\text { Span } \\
(\mathrm{mm})\end{array}$ & $\begin{array}{c}\text { Pmax } \\
(\mathrm{kN})\end{array}$ & $\begin{array}{c}\text { Deflection } \\
(\mathrm{mm})\end{array}$ & $\begin{array}{c}\sigma \text { max } \\
(\mathrm{MPa})\end{array}$ \\
\hline 12 & 12.00 & 113.10 & 240 & 2.135 & 16.03 & 755.1 \\
\hline 12 & 12.00 & 113.10 & 240 & 2.177 & 16.47 & 770.0 \\
\hline 12 & 12.00 & 113.10 & 240 & 2.209 & 17.64 & 781.3 \\
\hline 12 & 12.00 & 113.10 & 240 & 2.126 & 15.99 & 751.9 \\
\hline 12 & 12.00 & 113.10 & 240 & 2.547 & 18.26 & 900,8 \\
\hline 12 & 12.00 & 113.10 & 240 & 2.165 & 17.74 & 765.7 \\
\hline 12 & 12.00 & 113.10 & 240 & 2.132 & 17.25 & 754.0 \\
\hline 12 & 12.00 & 113,10 & 240 & 2.090 & 16.91 & 739.2 \\
\hline Average & 12.00 & & & 2.2 & & 775.0 \\
\hline Deviation & 0.00 & & & 0.08 & & 29.34 \\
\hline$\delta$ & $0.0 \%$ & & & $3.8 \%$ & & $3.8 \%$ \\
\hline Characteristic & 12.00 & & & 2.04 & & 720.45
\end{tabular}


Table 4. Bending test results for diameter $16 \mathrm{~mm}$

\begin{tabular}{c|c|c|c|c|c|c}
$\phi(\mathrm{mm})$ & $\phi$ real $(\mathrm{mm})$ & $\begin{array}{c}\text { Cross Area } \\
\left(\mathrm{mm}^{2}\right)\end{array}$ & $\begin{array}{c}\text { Span } \\
(\mathrm{mm})\end{array}$ & $\begin{array}{c}\text { Pmax } \\
(\mathrm{kN})\end{array}$ & $\begin{array}{c}\text { Deflection } \\
(\mathrm{mm})\end{array}$ & $\begin{array}{c}\sigma \text { max } \\
(\mathrm{MPa})\end{array}$ \\
\hline 16 & 16.00 & 201.06 & 320 & 3.985 & 19.97 & 792.8 \\
\hline 16 & 16.00 & 201.06 & 320 & 3.853 & 20.40 & 766.5 \\
\hline 16 & 16.00 & 201.06 & 320 & 4.099 & 21.94 & 815.5 \\
\hline 16 & 16.00 & 201.06 & 320 & 3.835 & 21.64 & 762.9 \\
\hline 16 & 16.00 & 201.06 & 320 & 3.688 & 20.87 & 733.7 \\
\hline 16 & 16.00 & 201.06 & 320 & 3.629 & 19.76 & 722.0 \\
\hline 16 & 16.00 & 201.06 & 320 & 3.452 & 18.45 & 686.8 \\
\hline 16 & 16.00 & 201.06 & 320 & 3.509 & 18.07 & 698.1 \\
\hline Average & 16.00 & & & 3.8 & & 757.6 \\
\hline Deviation & 0.00 & & & 0.21 & & 42.22 \\
\hline$\delta$ & $0.0 \%$ & & & $5.6 \%$ & & $5.6 \%$ \\
\hline Characteristic & 16.00 & & & 3.41 & & 679.10
\end{tabular}

Table 5. Bending test results for diameter $20 \mathrm{~mm}$

\begin{tabular}{c|c|c|c|c|c|c}
$\phi(\mathrm{mm})$ & $\phi$ real $(\mathrm{mm})$ & $\begin{array}{c}\text { Cross Area } \\
\left(\mathrm{mm}^{2}\right)\end{array}$ & $\begin{array}{c}\text { Span } \\
(\mathrm{mm})\end{array}$ & $\begin{array}{c}\text { Pmax } \\
(\mathrm{kN})\end{array}$ & $\begin{array}{c}\text { Deflection } \\
(\mathrm{mm})\end{array}$ & $\begin{array}{c}\sigma \mathrm{max} \\
(\mathrm{MPa})\end{array}$ \\
\hline 20 & 20.00 & 314.16 & 400 & 5.895 & 26.32 & 750.6 \\
\hline 20 & 20.00 & 314.16 & 400 & 5.694 & 25.87 & 725.0 \\
\hline 20 & 20.00 & 314.16 & 400 & 5.300 & 21.52 & 674.8 \\
\hline 20 & 20.00 & 314.16 & 400 & 5.694 & 23.67 & 725.0 \\
\hline 20 & 20.00 & 314.16 & 400 & 4.997 & 22.83 & 636.2 \\
\hline 20 & 20.00 & 314.16 & 400 & 5.772 & 24.17 & 734.9 \\
\hline Average & 20.00 & & & 5.6 & & 707.8 \\
\hline Deviation & 0.00 & & & 0,27 & & 34.82 \\
\hline$\delta$ & $0.0 \%$ & & & $4.9 \%$ & & $4.9 \%$ \\
\hline Characteristic & 20.00 & & & 5.01 & & 637.60
\end{tabular}

Table 6 . Bending test results for diameter $25 \mathrm{~mm}$

\begin{tabular}{c|c|c|c|c|c|c}
$\phi(\mathrm{mm})$ & $\phi$ real $(\mathrm{mm})$ & $\begin{array}{c}\text { Cross Area } \\
\left(\mathrm{mm}^{2}\right)\end{array}$ & $\begin{array}{c}\text { Span } \\
(\mathrm{mm})\end{array}$ & $\begin{array}{c}\text { Pmax } \\
(\mathrm{kN})\end{array}$ & $\begin{array}{c}\text { Deflection } \\
(\mathrm{mm})\end{array}$ & $\begin{array}{c}\sigma \mathrm{max} \\
(\mathrm{MPa})\end{array}$ \\
\hline 25 & 25.00 & 490.87 & 500 & 8.036 & 30.04 & 654.8 \\
\hline 25 & 25.00 & 490.87 & 500 & 7.751 & 30.66 & 631.6 \\
\hline 25 & 25.00 & 490.87 & 500 & 7.712 & 28.05 & 628.4 \\
\hline 25 & 25.00 & 490.87 & 500 & 7.745 & 29.38 & 631.1 \\
\hline 25 & 25.00 & 490.87 & 500 & 7.805 & 29.15 & 636.0 \\
\hline 25 & 25.00 & 490.87 & 500 & 7.563 & 27.32 & 616.3 \\
\hline Average & 25.00 & & & 7.8 & & 633.0 \\
\hline Deviation & 0.00 & & & 0.10 & & 8.25 \\
\hline$\delta$ & $0.0 \%$ & & & $1.3 \%$ & & $1.3 \%$ \\
\hline Characteristic & 25.00 & & & 7.56 & & 616.43
\end{tabular}


Table 7. Bending test results for diameter $32 \mathrm{~mm}$

\begin{tabular}{c|c|c|c|c|c|c}
$\phi(\mathrm{mm})$ & $\phi$ real $(\mathrm{mm})$ & $\begin{array}{c}\text { Cross Area } \\
\left(\mathrm{mm}^{2}\right)\end{array}$ & $\begin{array}{c}\text { Span } \\
(\mathrm{mm})\end{array}$ & $\begin{array}{c}\text { Pmax } \\
(\mathrm{kN})\end{array}$ & $\begin{array}{c}\text { Deflection } \\
(\mathrm{mm})\end{array}$ & $\begin{array}{c}\sigma \mathrm{max} \\
(\mathrm{MPa})\end{array}$ \\
\hline 32 & 32.00 & 804.25 & 640 & 13.439 & 38.22 & 668.4 \\
\hline 32 & 32.00 & 804.25 & 640 & 13.589 & 39.53 & 675.9 \\
\hline 32 & 32.00 & 804.25 & 640 & 13.820 & 38.78 & 687.4 \\
\hline 32 & 32.00 & 804.25 & 640 & 13.472 & 36.51 & 670,0 \\
\hline 32 & 32.00 & 804.25 & 640 & 14.329 & 39.70 & 712.7 \\
\hline Average & 32.00 & & & 13.8 & & 687.4 \\
\hline Deviation & 0.00 & & & 0.32 & & 15.96 \\
\hline$\delta$ & $0.0 \%$ & & & $2.3 \%$ & & $2.3 \%$ \\
\hline Characteristic: & 32.00 & & & 13.17 & & 655.22
\end{tabular}

\section{Comparison with pure tensile test}

In order to verify the validity of the proposed method, results obtained by means of bending test have been compared (Table 8) with those obtained in a pure tensile test [7] carried out on specimens from the same production batch.

Table 8. Tensile and bending test results classified according to diameter

\begin{tabular}{c|c|c|c}
$\phi(\mathrm{mm})$ & $\sigma_{\text {tensile }}(\mathrm{MPa})$ & $\sigma_{\text {bending }}(\mathrm{MPa})$ & Difference $(\%)$ \\
\hline 8 & 676.0 & 655.8 & $3.00 \%$ \\
\hline 10 & 684.1 & 644.4 & $5.81 \%$ \\
\hline 12 & 738.8 & 709.0 & $4.04 \%$ \\
\hline 16 & 816.4 & 658.7 & $19.32 \%$ \\
\hline 20 & 784.1 & 637.6 & $18.69 \%$ \\
\hline 25 & 752.8 & 616.4 & $18.11 \%$ \\
\hline 32 & 736.0 & 655.2 & $10.98 \%$ \\
\hline Average & 741.2 & 653.9 &
\end{tabular}

- Bending tests provides tensile strengths much more conservative than tensile test

- Within small diameters $(8,10$ and 12) the difference between tensile strength obtained by means of a bending test and tensile strength obtained by means of a pure tensile test is smaller than $6 \%$.

- Divergence of results increases as specimens diameter increases $(16,20,25$ and 32) reaching a maximum of $19.32 \%$ for specimens with a diameter of $16 \%$.

Results graphical representation (Figure 4) clearly shows that divergence with respect to strength arithmetic mean is smaller in the bending test.

\section{Conclusions}

This paper has shown the application of bending test in order to determine GFRP reinforcement bars tensile strength. 
When compared with results obtained by means of a pure tensile test it is obvious that this method provides conservative values. There is a good concordance of values for small diameters.

Bending tests is a simple and inexpensive test which allows determining GFRP rebars tensile strength with a sufficient accuracy and avoids complex tests specified in the code regulations.

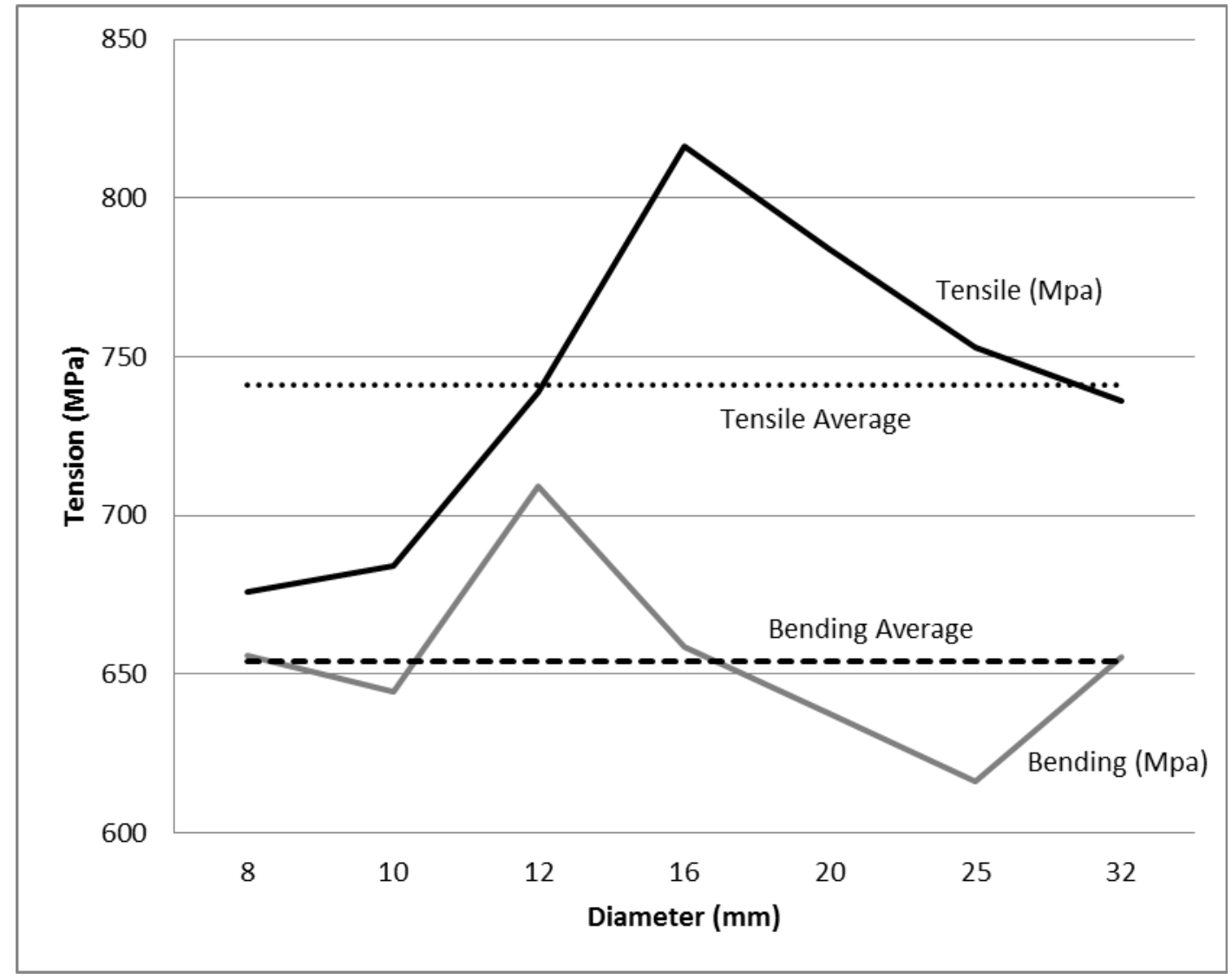

Figure 4. Results comparative

\section{References}

[1] A.I. Almerich Chulia. “Diseño, según Estados Limites, de Estructuras de Hormigon Armado Con Redondos de Fibra de Vidrio GFRP”. Thesis, Universidad Politecnica de Valencia, Valencia, Spain (2010).

[2] UNE 83306:1985 "Concrete tests. Indirect tension failure (Brazilian test)"

[3] UNE-EN 14651:2007+A1:2008. "Test method for concrete with metallic fibers. Tensile strength determining by means of bending test (Limit of Proportionality (LOP), residual strength)"

[4] UNE-EN ISO 178 "Plastics: Flexural properties determining"

[5] UNE-EN 13706-2:2003. "Reinforced plastics composites - Specifications for pultruded profiles - Part 2: Methods of test and general requirements"

[6] UNE 66040:2003 "Statistical interpretation of test results. Estimation of the mean. Confidence interval."

[7] A.I. Almerich-Chulia et al., "Innovative Design of GFRP Bars for Concrete Structures". Advanced Materials Research, 457-458, 553 (2012) 